\title{
Doing the wrong thing for the right reasons: The demise of the HVAD
}

\author{
Isabel Balachandran, MD, O. H. Frazier, MD, and Joseph G. Rogers, MD
}

Feature Editor's Introduction-The HeartWare HVAD System (Medtronic) was a durable left ventricular assist device (LVAD) approved for bridge to transplant and destination therapy indications and had been implanted in approximately 20,000 patients worldwide. The device gained wide adoption, particularly in clinical scenarios that used a left anterolateral thoracotomy approach for device placement, and for patients with smaller body size, and for off-label pediatric applications. In December 2020, Medtronic issued an Urgent Medical Device Communication informing clinicians of a critical device malfunction in which the HVAD System experienced either a delay or failure to restart following elective or accidental discontinuation of pump operation. In addition, mounting evidence from comparative effectiveness studies demonstrated a significantly greater risk of stroke and all-cause mortality in HVAD recipients compared with those receiving a newer generation of commercially available durable LVAD. Considering the totality of new information on HVAD performance, Medtronic and the US Food and Drug Administration halted its sale and distribution in June 2021.

In this issue of the Journal, Drs Balachandran, Frazier, and Rogers write a masterful commentary on the implications of the decision by Medtronic to halt the sale and distribution of the HVAD. While supporting this decision by Medtronic based on the issue of patient safety, the authors insightfully note that complete abandonment of the technology without a commitment to further research into reengineering of the device was perhaps premature and possibly unjustified. Given the long history of successful support by the device and limited choices for durable LVAD support now available to patients and clinicians, further investment into the device may have been warranted. As the authors note, the impact of this decision will hopefully spur a renewed sense of urgency from engineers and clinicians to collaboratively work to develop and bring to clinical use newer and safer devices.

\section{Francis D. Pagani, MD, PhD}

\footnotetext{
From the Texas Heart Institute, Houston, Tex.

Received for publication Aug 17, 2021; revisions received Sept 23, 2021; accepted for publication Oct 1, 2021; available ahead of print Nov 25, 2021.

Address for reprints: Joseph G. Rogers, MD, Texas Heart Institute, 6770 Bertner Ave, MC3-116, Houston, TX 77030 (E-mail: jrogers@texasheart.org). J Thorac Cardiovasc Surg 2022;164:1937-41 $0022-5223 / \$ 36.00$

Copyright (c) 2021 by The American Association for Thoracic Surgery https://doi.org/10.1016/j.jtcvs.2021.10.058
}

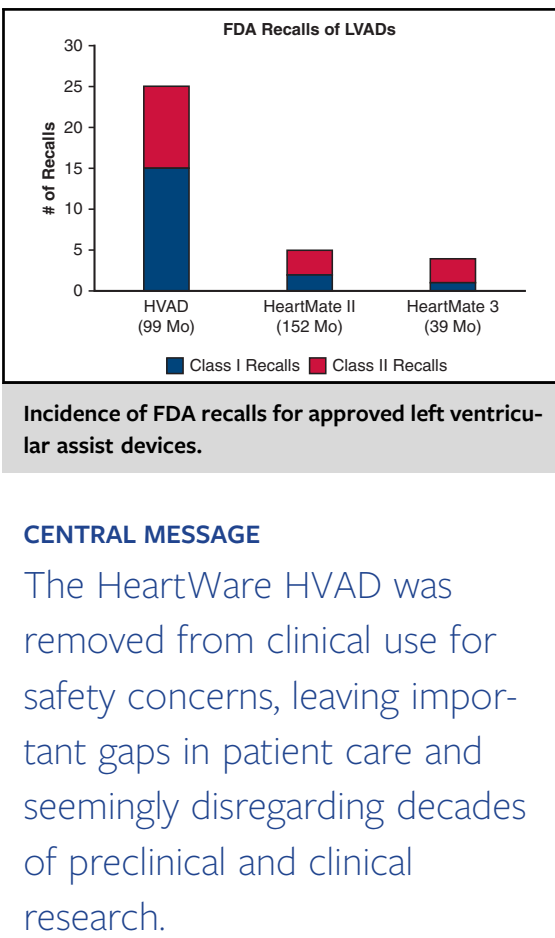

This Invited Expert Opinion provides a perspective on the following papers: Circulation. 2021 Aug 17;144(7):509-511. CIRCULATIONAHA.121.056027 and Heart Lung Transplant. 2021 May;40(5):323-333. https:/doi org/10.1016/j.healun.2021.02.010

See Commentaries on pages 1942 and 1943.

The development of durable continuous-flow left ventricular assist devices (LVADs) has revolutionized the field of heart failure, providing meaningful improvements in longevity and quality of life for patients with otherwiseterminal heart disease regardless of their candidacy for cardiac transplantation. Following years of preclinical and clinical research, 3 devices achieved the critical milestone of Food and Drug Administration (FDA) approval for use in patients: HeartMate II, HeartMate 3 (both Abbott) and HeartWare HVAD (Medtronic). In controlled clinical trials, each of these devices was effective in supporting patients for periods up to 24 months with improvements in survival, quality of life, and functional status with acceptable adverse event profiles relative to comparator therapies. The 
sequential nature of the trials allowed patients and clinicians to carefully consider the advantages and disadvantages of the individual devices, tailor the pump to the patient, and participate in a shared decision-making construct.

The US HeartWare HVAD clinical trials program carefully studied more than 900 patients with advanced heart failure supported with the device between 2008 and $2016{ }^{1-4}$ The bridge-to-transplant and destination therapy (DT) trials tested the hypothesis that survival without disabling stroke or the need to replace the HVAD would be noninferior to contemporaneous, commercially available LVADs. Component analysis of the primary end point in the DT trial demonstrated that HVAD survival was similar to HeartMate II and associated with nearly a 50\% reduction in device failure/malfunction rates. However, a concerning safety signal emerged in the DT trial - the stroke rate with HVAD was significantly greater than seen with HeartMate II $(29.7 \%$ vs $12.1 \%, P<.001)$. A retrospective analysis of the clinical trials datasets suggested that patients with relative hypertension were at greatest risk of stroke, prompting the ENDURANCE Supplemental Trial to test the hypothesis that a standardized approach to blood pressure measurement in patients with nonpulsatile flow and focused blood pressure control would lower the stroke risk in patients with an HVAD. ${ }^{4,5}$ In that study, improved blood pressure control coupled with greater attention to anticoagulation and antiplatelet therapy reduced neurologic events primarily by decreasing hemorrhagic stroke $(P=\mathrm{NS})$. As a result, clinicians managing patients with an HVAD were encouraged to maintain systemic arterial blood pressure $\leq 90 \mathrm{~mm} \mathrm{Hg}$ targeting the first audible sound on Doppler.

More than 19,000 HVADs were implanted over the lifecycle of the device. The clinical utility of the device was related to its design as a magnetically levitated centrifugal flow pump containing only one hydrodynamic bearing, intrapericardial implantation eliminating the need for a pump pocket, enhanced monitoring capabilities that provided unique physiological insights, and a smaller size that allowed implantation in small adults and children with excellent results as well as off-label use to support the failing right ventricle. ${ }^{6-8}$ The HVAD inflow cannula is integrated into the central pump housing, lending itself to diaphragmatic placement, a position that was infrequently used clinically but mitigated the challenge of cannula alignment in the long axis of the left ventricle and facilitated use in narrow-chested patients with marked cardiomegaly. ${ }^{9}$ HVAD was also subjected to a nonrandomized clinical study of implantation through a less-invasive approach using a limited anterolateral thoracotomy combined with an upper hemisternotomy or right anterior thoracotomy for attachment of the outflow graft to the aorta. Over a 6-month follow-up period,
$88 \%$ of patients were alive on the original device and free from a disabling stroke, a rate greater than anticipated based on historical data. ${ }^{10}$

On June 3, 2021, Medtronic and the FDA abruptly announced that production and distribution of the HVAD would be stopped permanently. While the complications related to HVAD were recognized and acknowledged, the announcement took many by surprise and resulted in clinician confusion and patient angst. Several factors were cited in the decision to discontinue HVAD, including an increase in the number of significant device malfunction reports, the stroke rate, and the evolution of HeartMate 3 as the preferred device used by implanting centers.

Clinicians and investigators involved in mechanically assisted circulation understand and appreciate the complexity and barriers to successful patient outcomes. LVADs are arguably some of the greatest risk of all medical devices resulting from the requirement for surgical implantation in a moribund patient cohort, the design and mechanics of the devices and controller systems, the physiologic alterations introduced into the systemic circulation by nonpulsatile blood pumps, and the need for lifelong, imperfect anticoagulation with warfarin to improve hemocompatibility. The device is also subjected to interaction with the patient and the vicissitudes of daily life, including physical activity, changing power supplies, and manipulation of the driveline. The interplay of these factors predisposes patients with continuous-flow LVAD to the potential for significant adverse events.

The development cycle of circulatory assist devices is long and laborious. One of us (O.H.F.) has been investigating and iteratively improving continuous flow LVADs since 1985. Following preclinical testing, HVAD was subjected to well-designed and rigorous clinical trials wherein investigators carefully studied and documented device performance, adverse events, and patient outcomes. The FDA approved the device in 2012 based on controlled studies demonstrating that HVAD was safe and effective for the treatment of the intended patient cohort. Following approval, FDA device surveillance occurred via a mandatory reporting mechanism that allowed the agency to oversee and mandate corrective action based on emerging events and trends that posed significant safety risks to patients. The recall process (Table 1) is a signal to the manufacturer, clinicians, and the public that safety thresholds have been crossed. It is through this lens that the decision to halt production and clinical application of HVAD becomes clearer.

Between May 2013 and June 2021, HVAD was the subject of 25 FDA recalls, $60 \%$ of which were classified as Class I and the remainder Class II. The incidence of HVAD recalls was 5-fold that of HeartMate II and increased significantly following the acquisition of HeartWare by Medtronic (Figure 1). ${ }^{11}$ The FDA received more than 100 reports of HVAD malfunction contributing to 27 instances 
TABLE 1. Classes included in the recall process of the Food and Drug Administration

\begin{tabular}{ll}
\hline $\begin{array}{c}\text { Type of } \\
\text { Recall }\end{array}$ & \multicolumn{1}{c}{ Definition } \\
\hline Class I & Recall of products that could cause serious injury or death \\
Class II & Recall of products that might cause serious injury or death \\
Class III & Recall of products unlikely to cause serious injury or death \\
\hline
\end{tabular}

of death or the need for pump replacement. Notable issues included electric discharges leading to pump failure, malfunctions of the pump controller, and numerous issues relating to adapter cables and batteries. ${ }^{12}$ Further, there were 29 cases in which the device had delayed or complete failure to restart, contributing to 2 deaths.

Even though these safety concerns arose after more than 13 years of widespread clinical acceptance and use, a decision to pause the clinical use of HVAD is prudent. However, the decision to abandon HVAD seems a premature and unjustified choice. It discounts years of thoughtful engineering, clinical study, and, most importantly, the commitments and sacrifices made by patients and their families in the clinical trials. The decision also runs counter to the cycle of innovation-invent, study, refine, study.

So why totally abandon HVAD at this time? Although there are no prospective, comparative clinical trials of HVAD and HeartMate 3, there is a growing body of evidence that clinicians favor the more recently introduced device. Numan and colleagues ${ }^{13}$ analyzed a propensitymatched cohort of patients with Heartmate 3 and HVAD and found no difference in survival but greater rates of pump thrombosis and hemorrhagic stroke with HVAD. Analyses of the Intermacs (Interagency Registry for Mechanically Assisted Circulatory Support) registry using propensity-matched populations implanted between 2017 and 2019 have also recently demonstrated that HVAD is associated with a 5- to 6-fold increase in stroke and a 3.2fold increase in mortality relative to HeartMate $3 .{ }^{14,15}$ The Intermacs 2020 annual report frames the US perceptions of the 2 devices. Following completion and publication of the MOMENTUM (Multicenter Study of MagLev Technology in Patients Undergoing Mechanical Circulatory Support with HeartMate 3) trial, HeartMate 3 has assumed a dominant position in the LVAD market (Figure 2) ${ }^{16}$ Thus, despite a lack of head-to-head clinical data supporting the use of HeartMate 3, US cardiologists, surgeons, and patients appear to be convinced of the superiority of HeartMate 3. Finally, and importantly, economic analysts have stated that HVAD is unprofitable for Medtronic, likely related to the decline in clinical implants.

Medtronic's decision to halt production and implantation of HVAD can be viewed from 2 distinct perspectives. The company's foundational commitment to providing safe and effective therapies is laudable and shared by the clinicians and patients. The totality of data suggests that HVAD reliability and adverse event profiles are placing patients at risk for negative outcomes. Further, an alternative, commercially available device may be superior and physicians are "voting with their feet," as evidenced by the declining number of HVADs implanted in the past few years. Medtronic had the right reasons to halt HVAD implants at this time. However, it should be argued that Medtronic's decision to entirely abandon a device that has been in clinical use in the United States since 2008 and FDA approved for nearly a decade is wrong. A more grounded approach would have been to return the device to a re-engineering phase to develop a deep understanding of the problems and subsequent mitigation strategies. Their decision leaves critical gaps for the 4000 patients currently on HVAD support and other vulnerable heart failure populations who no longer have a viable option for durable LVAD support, including children and those with right heart failure.

Temporal FDA Recalls of HVAD

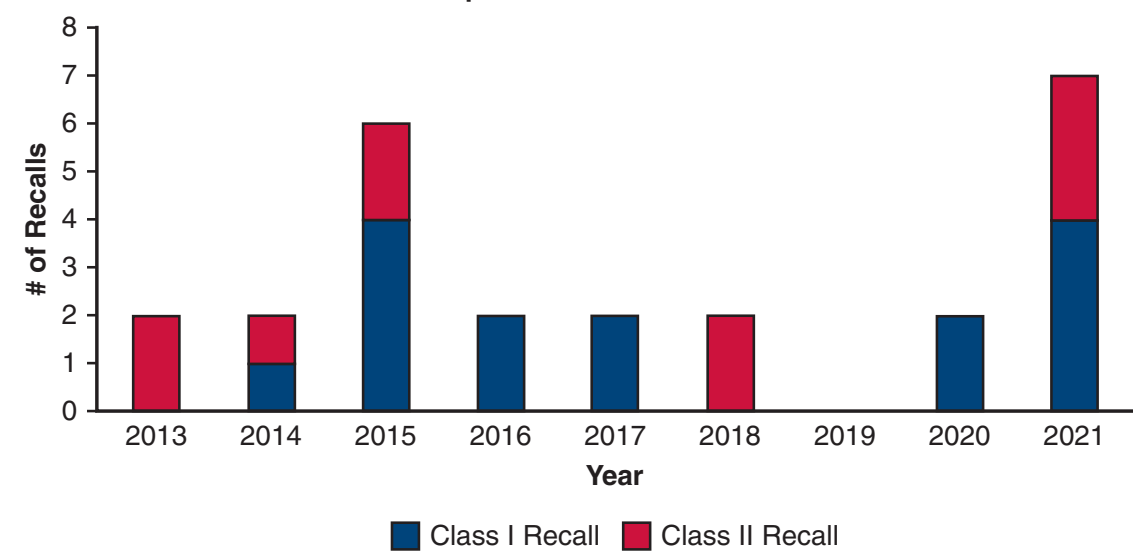

FIGURE 1. Temporal trends in HVAD recalls, 2013 to $2021 .{ }^{11}$ FDA, Food and Drug Administration. 


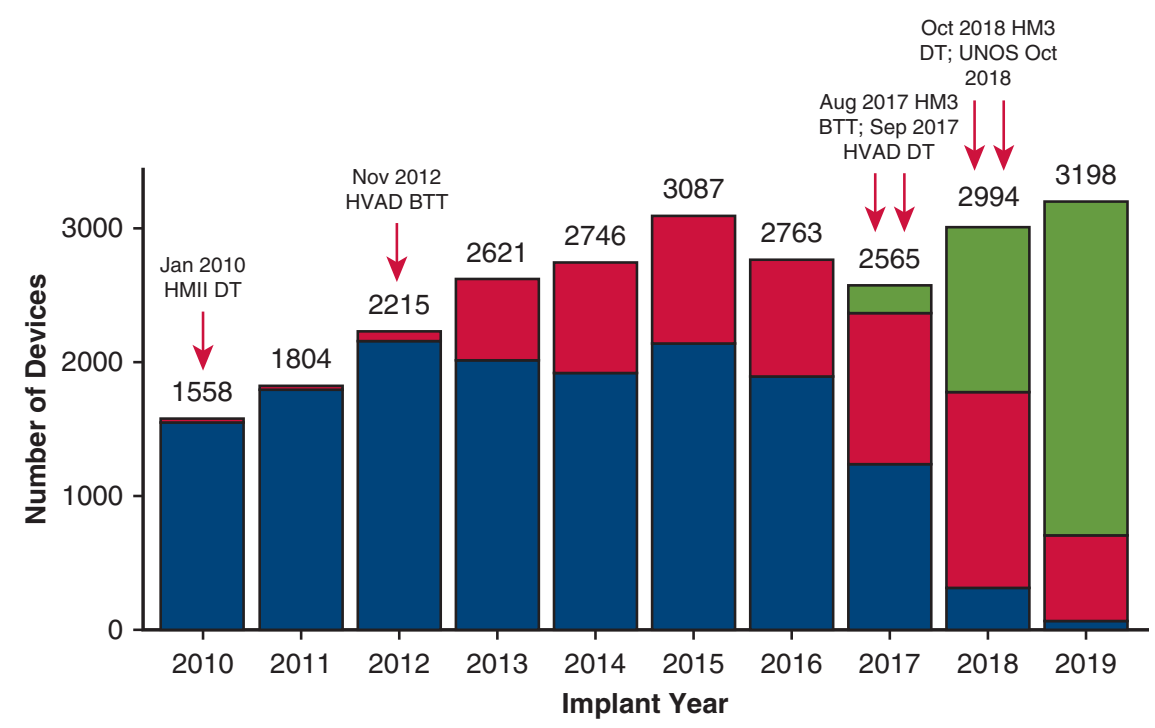

Pump Group $\square$ CFLVAD axial $\square$ CFLVAD hybrid $\square$ CFLVAD maglev

FIGURE 2. Device implantation by year reported to the Intermacs database. CFLVAD axial represents the HeartMate II, CFLVAD hybrid represents HeartWare HVAD, and CFLVAD maglev represents HeartMate 3. From Molina and colleagues. ${ }^{16} H M I I$, HeartMate II; DT, destination therapy; BTT, bridge to transplant; HM3, HeartMate 3; UNOS, United Network for Organ Sharing; CFLVAD, continuous-flow left ventricular assist device.

The clinical community's hope for acquisition of small LVAD companies by Abbott (Thoratec) and Medtronic (HeartWare) was incremental investment in research and development to improve device reliability, safety, and function. While there are several mechanical circulatory support devices in various phases of developmental programs, we are currently left with one commercially available, durable device. While the literature demonstrates a complex relationship between competition and innovation, concerns abound regarding incremental investments to improve HeartMate 3.

With current advances in technology and innovation, there is a unique opportunity to channel the lessons learned from the HVAD experience to develop safer and more effective durable LVADs in the future. The field of assisted circulation has a rich history of collaborative science and patient engagement. Clinical scientists, bioengineers, and patients have worked together to develop and optimize durable circulatory support devices with full recognition of the challenges and risks. Going forward, clinicians, investigators, patients, industry partners, and regulators must continue to innovate, iterate, and investigate to achieve the ultimate goals of heart-replacement therapy.

\section{Conflict of Interest Statement}

Dr Frazier reports consultant for Abiomed and receives related travel reimbursement for speaking engagements. Dr Frazier also receives related travel reimbursement from BiVACOR to attend Clinical Advisory Board meetings, on which he also serves. All other authors reported no conflicts of interest.

The Journal policy requires editors and reviewers to disclose conflicts of interest and to decline handling or reviewing manuscripts for which they may have a conflict of interest. The editors and reviewers of this article have no conflicts of interest.

\section{References}

1. Aaronson KD, Slaughter MS, Miller LW, McGee EC, Cotts WG, Acker MA, et al. Use of an intrapericardial, continuous-flow, centrifugal pump in patients awaiting heart transplantation. Circulation. 2012;125:3191-200.

2. Slaughter MS, Pagani FD, McGee EC, Birks EJ, Icenogle T, Najjar SS, et al. HeartWare ventricular assist system for bridge to transplant: combined results of the bridge to transplant and continued access protocol trial. J Heart Lung Transplant. 2013;32:675-83.

3. Rogers JG, Pagani FD, Tatooles AJ, Bhatt G, Slaughter MS, Birks EJ, et al. Intrapericardial left ventricular assist device for advanced heart failure. $N$ Engl J Med. 2017;376:451-60.

4. Milano CA, Rogers JG, Tatooles AJ, Bhatt G, Slaughter MS, Birks EJ, et al. HVAD: the ENDURANCE supplemental trial. JACC Heart Fail. 2018;6: 792-802.

5. Teuteberg JJ, Slaughter MS, Rogers JG, McGee EC, Pagani FD, Gordon R, et al. The HVAD left ventricular assist device. Risk factors for neurological events and risk mitigation strategies. JACC Heart Fail. 2015;3:818-28.

6. VanderPluym CJ, Adachi I, Niebler R, Griffiths E, Flynn-Thompson F, Chen S. Outcomes of children supported with an intracorporeal continuous-flow left ventricular assist system. J Heart Lung Transplant. 2018;38:385-93.

7. Spigel ZA, Cho J, Adachi I. Current status of pediatric mechanical circulatory support. Curr Opin Organ Transplant. 2020;25:231-6.

8. Marasco A, Simon AR, Tsui T, Schramm R, Eifert S, Hagl CM, et al. International experience using a durable, centrifugal-flow ventricular assist device for biventricular support. J Heart Lung Transplant. 2020;39:1372-9.

9. Gregoric ID, Cohn WE, Frazier OH. Diaphragmatic implantation of the HeartWare ventricular assist device. J Heart Lung Transplant. 2011;30: 467-70. 
10. McGee E, Danter M, Strueber M, Mahr C, Mokadam NA, Wieselthaler G, et al. Evaluation of a lateral thoracotomy implant approach for a centrifugal-flow left ventricular assist device: the LATERAL clinical trial. J Heart Lung Transplant. 2019:38:344-51.

11. US Food and Drug Administration. Medical device recalls. Accessed August 14, 2021. https://www.accessdata.fda.gov/scripts/cdrh/cfdocs/cfRES/res.cfm

12. Kuehn BM. FDA: stop using Medtronic's HeartWare ventricular assist device. JAMA. 2021;326:215.

13. Numan L, Ramjankhan FZ, Oberski DL, Oerlemans MIFJ, Aarts E, Gianoli M, et al. Propensity score-based analysis of long-term outcome of patients on HeartWare and HeartMate 3 left ventricular assist device support. ESC Heart Fail. 2021;8:1596-603.

14. Cho SM, Mehaffey JH, Meyers SL, Cantor RS, Starling RC, Kirklin JK, et al. Cerebrovascular events in patients with centrifugal-flow left ventricular assist devices: a propensity score matched analysis from the Intermacs Registry. $\mathrm{Circu}$ lation. 2021;144:763-72.

15. Pagani FD, Cantor R, Cowger J, Goldstein DJ, Teuteberg JJ, Mahr CW, et al. Concordance of treatment effect: an analysis of the Society of Thoracic Surgeons Intermacs database. Ann Thorac Surg. June 1, 2021 [Epub ahead of print]. https:// doi.org/10.1016/j.athoracsur.2021.05.017

16. Molina EJ, Shah P, Kiernan MS, Cornwell WK III, Copeland H, Takeda K, et al. The Society of Thoracic Surgeons Intermacs 2020 annual report. Ann Thorac Surg. 2021;111:778-92.

Key Words: mechanically assisted circulation, LVAD, heart failure 